\title{
Redesigning a Department of Surgery during the COVID-19 Pandemic
}

\author{
Stacy A. Brethauer ${ }^{1} \cdot$ Benjamin K. Poulose ${ }^{1} \cdot$ Bradley J. Needleman ${ }^{1} \cdot$ Carrie Sims $^{1} \cdot$ Mark Arnold $^{1}$. \\ Kenneth Washburn ${ }^{1} \cdot$ Allan Tsung $^{1} \cdot$ Nahush Mokadam $^{1} \cdot$ Timur Sarac $^{1} \cdot$ Robert Merritt $^{1} \cdot$ Timothy M. Pawlik $^{1,2}$
}

Received: 7 April 2020 / Accepted: 8 April 2020 / Published online: 28 April 2020

(C) 2020 The Society for Surgery of the Alimentary Tract

\begin{abstract}
Background COVID-19 has created an urgent need for reorganization and surge planning among departments of surgery across the USA.

Methods Review of the COVID-19 planning process and work products in preparation for a patient surge. Organizational and process changes, workflow redesign, and communication plans are presented.

Results The planning process included widespread collaboration among leadership from many disciplines. The department of surgery played a leading role in establishing clinical protocols, guidelines, and policies in preparation for a surge of COVID-19 patients. A multidisciplinary approach with input from clinical and nonclinical stakeholders is critical to successful crisis planning. A clear communication plan should be implemented early and input from trainees, staff, and faculty should be solicited.

Conclusion Major departmental and health system reorganization is required to adapt academic surgical practices to a widespread crisis. Surgical leadership, innovation, and flexibility are critical to successful planning and implementation.
\end{abstract}

Keywords COVID-19 $\cdot$ Surgery $\cdot$ Planning $\cdot$ Leadership $\cdot$ Communication $\cdot$ Surge

\section{Introduction}

The COVID-19 pandemic has created unprecedented challenges for surgery departments throughout the country. Swift planning and strong leadership have been required to prepare for this crisis. The challenges are especially dire as no situation in recent memory has had such an impact on healthcare in the USA. We describe our department of surgery preparation for an anticipated COVID-19 surge. The strategies highlighted here address the challenges we faced in operational and clinical restructuring, communication plans, the educational and training mission, and resource management.

Timothy M. Pawlik

tim.pawlik@osumc.edu

1 Department of Surgery, Divisions of General and Gastrointestinal Surgery and Surgical Oncology, The Ohio State University, Columbus, $\mathrm{OH}$, USA

2 The Ohio State University Wexner Medical Center, 395 W. $12^{\text {th }}$ Avenue, Faculty Office Tower $6^{\text {th }}$ Floor, Columbus, OH 43210-1267, USA

\section{Background}

The first cluster of novel coronavirus cases was identified in Wuhan, China, in December 2019, and the virus was subsequently named by the World Health Organization (WHO) as COVID-19 in February of 2020 (Figure 1). ${ }^{1}$ After the first reports from China, the virus quickly spread globally, and the first case in the USA was reported in January 2020 in Washington State. ${ }^{2}$ At the time of this writing, there are over 350,000 cases and over 10,000 deaths related to COVID-19 in the USA. A national emergency was declared in the USA on March 13, 1 day after the WHO declared the coronavirus a pandemic.

This rapidly changing situation resulted in dramatic changes in the way health systems needed to function. Normal hospital operations were significantly reduced by postponing all non-essential procedures. Statewide "stay-at-home" orders required radical changes triaging personnel to be present at hospital facilities and those who may work remotely from home. Simultaneously, enormous efforts were made to stockpile personal protective equipment (PPE) and to establish policies regarding its use. Within the department of surgery, these challenges required leadership to form new workgroups and reporting structures, establish clear communication strategies, 


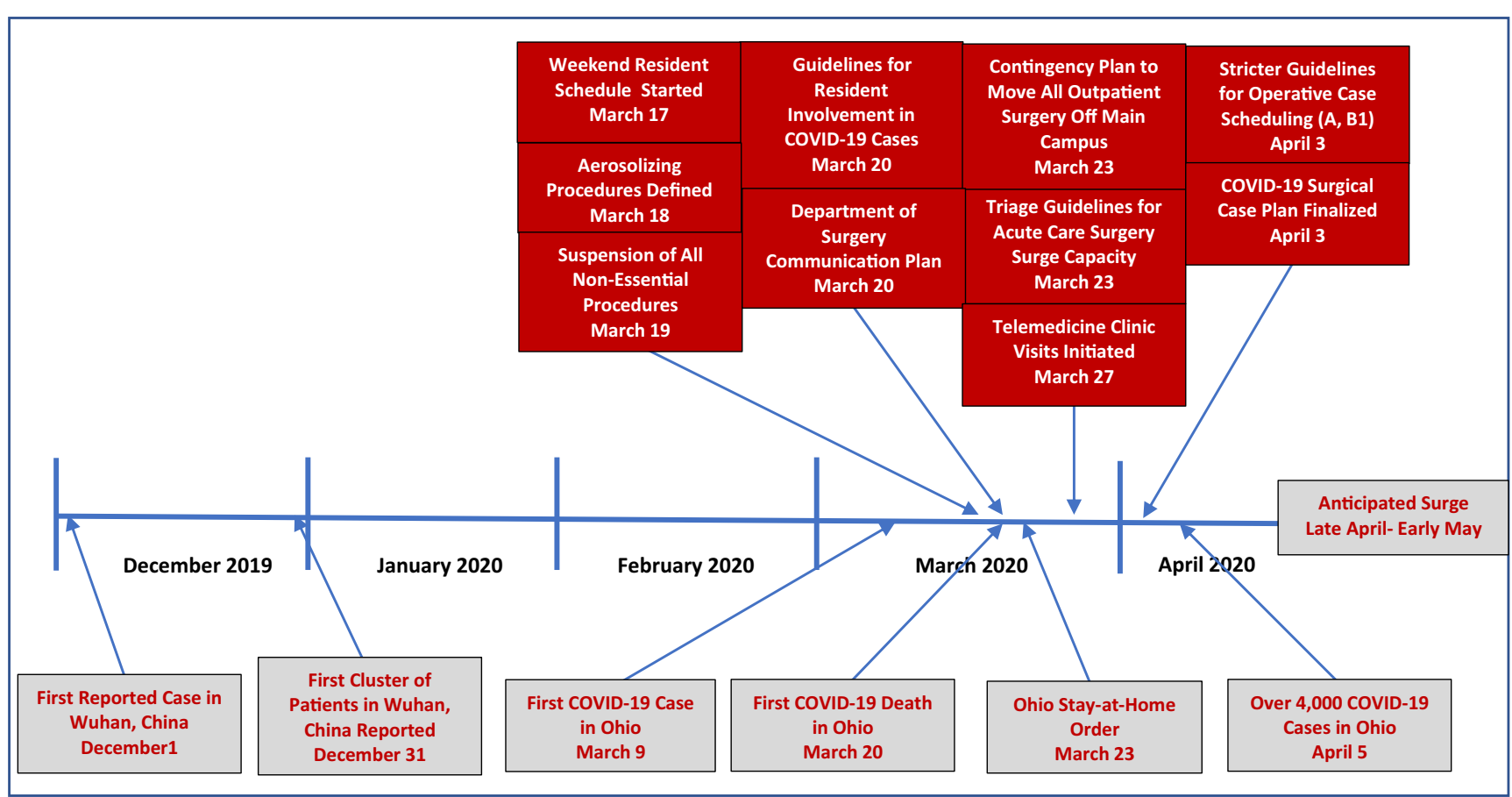

Fig. 1 Timeline of COVID-19 pre-surge planning

redefine clinical activities for the faculty, and modify the workloads of trainees. As the pandemic continues, it will be imperative that initial plans and strategies presented here be continuously evaluated and modified to address the fluidity of the situation.

\section{Information Sources}

Making evidence-based decisions as a crisis develops and evolves is difficult. Information changes daily, data can be unreliable, and new assumptions are required as the scenario progresses. Nevertheless, it is important to understand where the most reliable sources of data exist, both within and outside the health system.

Within the health system, the faculty and staff should expect regular updates on the crisis and new policies that affect their practices. Internal data will come from a variety of sources including the C-suite and University leadership, health system analysts, and a variety of workgroups established specifically for the hospital incident command. The workgroups that were established to prepare for the COVID-19 crisis at our institution are shown in Table 1. Appropriate surgical representation is needed within these workgroups. Personnel chosen for these roles should play an active part in task management, communication, and innovation. Appealing to the genuine call for service to the hospital community is critical as these roles cannot be seen as the stereotypical passive "committee member" role.
Data sources outside the health system included the Centers for Disease Control, the Ohio Department of Health, the Ohio Hospital Association, and information from our affiliate hospitals and network of clinics. ${ }^{3}$ Ohio was one of the first states to implement social distancing and stay-at-home policies, and this information was provided by the governor's office with regular updates to the medical center. These policies resulted in immediate and major changes in workforce planning, staffing, hospital operations, and clinical schedules.

Predicting the number of patients during the expected COVID-19 surge is difficult and has been based on both internal and external data. The Ohio Department of Health provided

Table 1 Preparation workgroups established by the hospital incident command

Health of workforce (faculty and staff self-monitoring)*

Covid19 call center workgroup

Swabbing/triage stations

Clinical care and testing workgroup*

Entrance visitor/public screening and public spaces workgroup

Labor pool workgroup

Supply chain*

Elective Surgical/procedural/ambulatory visits*

Telehealth

Alternative sites of care workgroup

Resource allocation (transfers, beds, vents, etc.)

Work from home workgroup

*Department of surgery representation 
updates based on modeling from a variety of sources and data regarding the virus activity throughout the state. Analysts focused on predictive models for our state and county to estimate what our health system would encounter (Figure 2). These data were used to model the number of beds, ventilators, personnel, and PPE that will be required for a surge of that magnitude.

\section{Shifting the Workforce}

The decision to suspend all elective and non-essential procedures was a major disruptor to the medical center and the faculty practices. Anticipation of such a policy prompted the departmental leadership to define the type of cases that would continue early in the course of the crisis. Once the policy was implemented, each division had already decided on a finite list of procedures or indications to perform operations based on the more general guidelines provided by the medical center, state, and professional societies. The perioperative leadership for each hospital pavilion was responsible for reviewing the operative case lists 2 days prior and escalating any questionably non-essential cases to the attending surgeon and then to the surgeon-in-chief if needed. In general, the policy was implemented in a way that respected the faculty's clinical decision regarding the consequences of postponement.

The surgical leadership across the health system established patient categories to prioritize procedures based on the urgency (Table 2). As the pandemic progresses, there are many factors that will impact the ability to perform surgery. Day-to-day, datadriven risk-benefit analyses must influence care delivery and case triage. Avoidance of blanket policies in lieu of careful and frequent analysis of the multiple factors is needed. These factors include operating room capacity, bed availability, equipment, supplies and PPE availability, ventilator availability, staffing, blood availability, and assessment of the national, regional, and local conditions and trends that may affect the magnitude of the surge. Two weeks after the initial policy was implemented, guidance was given to tighten the window for essential cases from 3 to 1 month based on modeling for the surge and limited resources. We also established different threat levels to the health system to guide our surgical case scheduling (Table 3).

Each clinical division developed a coverage schedule that minimized the number of faculty coming to work every day. Each division in the department of surgery was given the

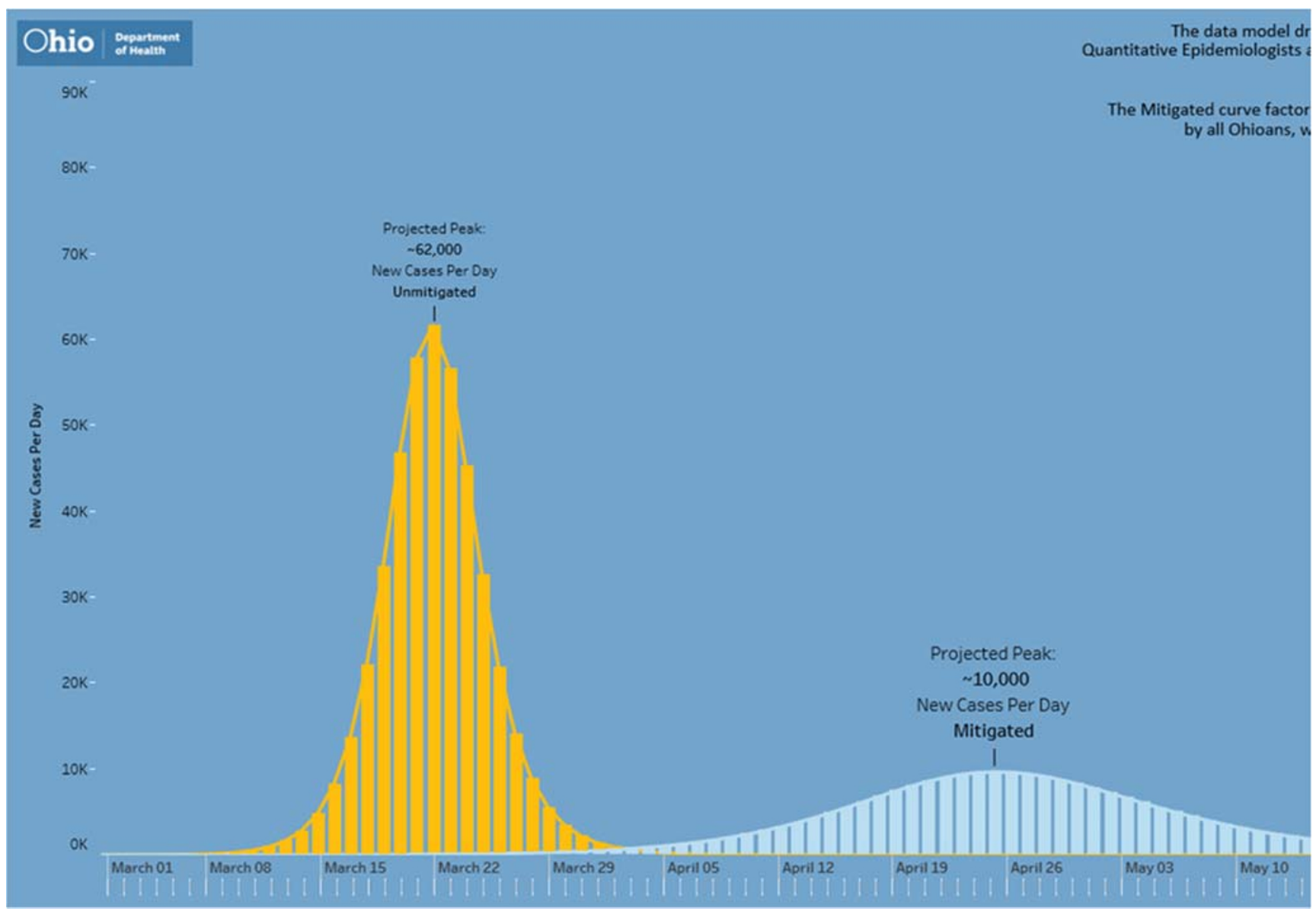

Fig. 2 Surge prediction for the Ohio State University. OSUWMC, Ohio State University Wexner Medical Center 
Table 2 Covid-19 procedure prioritization plan

Patient experiencing acute conditions that require urgent or emergent
surgical treatment, whose care cannot be delayed or deferred
Examples may include:
- Perforations/fistulae (bowel, esophageal)
- Obstruction (bowel, urinary tract, airway)
- Trauma (MVA, GSW, etc.)
$\cdot$ Necrotizing infections or surgically treatable infections
with signs of sepsis
Spinal cord compression requiring neuro release
Patient deemed to be in a non-life threatening status, whose surgery
could safely be deferred for a short period of time (<3 months).
Surgeons will determine that these patients are not put at undue risk.
If their priority changes, they will be moved to category A. Patients
can be categorized in the following sub-groups
B1: Case can be deferred no more than 1 month
B2: Case can be deferred $2-3$ months
Patient whose evaluation or treatment can safely be delayed
for an extended period ( $>3$ months). These patients are
for the most part non-essential cases that can reasonably wait until
the pandemic is over

$M V A$, motor vehicle accident; $G S W$, gunshot wound latitude to create their own coverage policy. For services with no elective cases, a single attending typically rounded on the service each day. For services still performing some essential operations (e.g., advanced cancer, emergent cardiac, trauma, or acute care surgery), every effort was made to minimize the number of faculty coming to work with coverage provided by those who were coming in for operative cases or on call.

Delivery of outpatient care was dramatically altered as well. As social distancing policies were implemented, in-person clinic appointments were limited to only those patients whose visit was deemed essential by the attending surgeon. Clinic staffing was evaluated and reduced to the minimum number of providers needed to see scheduled or urgent add-on patients. Telemedicine was implemented across the system, and consults and follow-up visits were transitioned as individually appropriate. Two following modalities of telemedicine were utilized: telephone based visits or video visits. An unintended, but positive, consequence of this rapid shift and adoption of
Table 3 Threat levels to guide surgical case scheduling during COVID-19
Level 0 - No threat

Level 1-Minimal threat:

Level 2-Moderate threat:

Level 3-Serious threat:

Level $4-$ Severe threat:

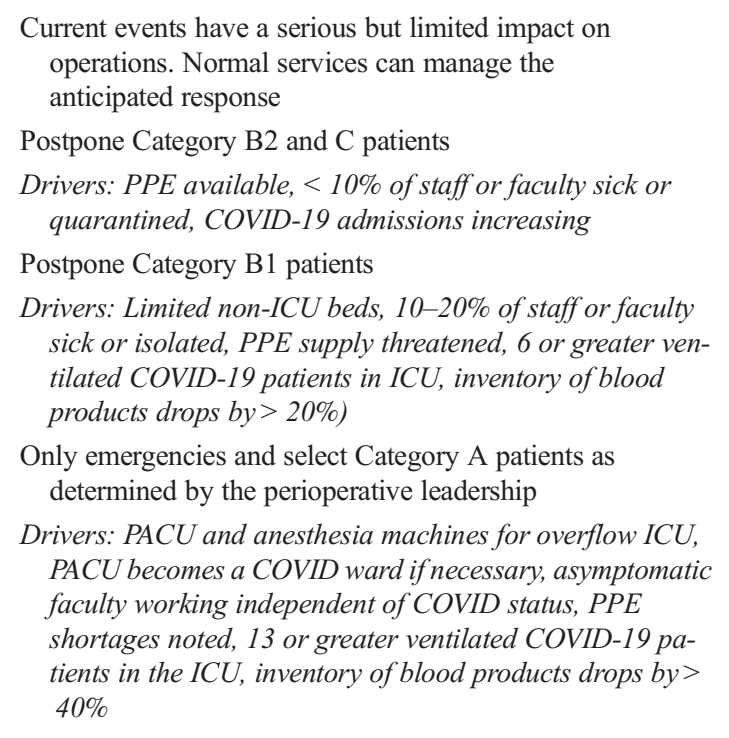

$P P E$, personal protective equipment; $I C U$, intensive care unit; $P A C U$, postoperative anesthesia care unit 
telemedicine forced the institution to quickly develop protocols and assistance with telemedicine operations, billing, workflow, compliance, information technology, and equipment. The shift was so rapid due to necessity that regulatory and compliance guidance was often lacking or very difficult to find.

\section{Redeploying Surgeons}

Within the department of surgery, a needs assessment was completed to formulate an acute care surgery surge capacity plan, recognizing that those currently serving in this capacity may be needed for their intensivist skillset. A call for volunteers to cover the ICU, trauma, or acute care surgery services was sent to faculty members outside the division covering trauma and acute care surgery and the surgical intensive care units. Specifically, surgeons were asked about their comfort level and privileging to manage critically ill patients and take emergency general surgery or trauma call. When the surveys were returned, surgeons were placed into different tiers to be called upon when needed during the surge. Tier 1 represented those who were comfortable with these skills and were currently credentialed to perform them. Tier 2 represented a pool of surgeons who were reasonably comfortable with ventilator management and ICU care but were not currently credentialed to do so, and Tier 3 represented the pool of surgeon who were not comfortable with these skills and were not credentialed to perform them. Only those individuals with current ATLS certification were placed in the trauma call pool.

Training was then initiated by the trauma and critical care staff to provide refresher training to those who would potentially be redeployed to the ICU. These training sessions were conducted via videoconference and included presentations on ventilator management, critical care for the non-intensivist, pharmacotherapy, and modules from the society of critical care medicine's "ICU Care for the Non-ICU Physician". It was important to communicate to the faculty, to alleviate anxiety and provide the safest care, that if they are to be deployed to the intensive care unit that there would always be an intensivist available to provide oversight and assistance throughout their assignment.

Prior to the surge, the faculty were asked to refocus their time on nonclinical activities related to career development and scholarly pursuits (Table 4). Importantly, the department established a COVID-19 Wellness Team that organized virtual social and entertainment activities with the staff and residents during this trying and stressful time.

\section{Communication Plan}

Clear, accurate, and frequent two-way communication is necessary in times of crisis. A situation like COVID-19 presents many communication challenges including a dynamic situation, simultaneous development of multiple policies, uncertainty about the scale of the future surge, and conflicting information on the same topic.

The department chair is in the best position to relay messages from multiple sources above and below them in the chain of command. The cadence and meeting composition will differ depending on the size and scope of each department, but we established weekly or bi-weekly calls with our division chiefs, department executive committee, and the perioperative cabinet (Table 5). Additionally, the chair held open virtual "Chair Hours" once a week for people to ask questions are express concerns. An "All Hands" call was held every other week for all of the clinical (faculty, trainees, mid-level providers) and administrative personnel that provided key updates and allowed for a town hall type of interaction. The presenters at these informational meetings included members of various work groups, supply chain, epidemiology, infectious disease, and senior leadership for the system. Of note, it has been critical that leadership informs attendees at every meeting that the data presented on that day represented our current knowledge and may change tomorrow. Setting this expectation with the faculty alleviates some of the angst and frustration of conflicting or changing messages. Finally, the residency program director and chairman held a weekly "Happy Hour" with all of the residents to get their feedback and address their needs.

\section{Education and Training Plan}

To protect the health of the trainees and preserve the workforce, our residency schedule was changed to a holiday schedule in which only half of the residents cover the services for blocks of several days. Fellows were incorporated into the faculty rounding and call coverage whenever credentialing allowed. Plans were developed for deployment of trainees to areas of critical need during the surge. Education conferences continued virtually including morbidity and mortality conference and grand rounds. Specific guidance for how residents should interact with COVID-19 patients was provided as follows:

\section{Phase I: Initial influx of COVID-19 patients Residents shall:}

a. Defer evaluation and consultation of COVID-19 positive patients to a faculty member.

i. Be physically present outside the isolation area and be prepared to assist if the faculty member requires immediate assistance (e.g., Level 2 or 3 trauma activation).

b. Perform evaluation and consultation of COVID-19 positive patients simultaneously with a faculty 
Table 4 Department faculty development plan during COVID-19 crisis

OSU department of surgery

Faculty activities/development during the COVID-19 pandemic

With normal work activities and assignments being altered during the OSU and DOS response to the COVID-19 pandemic, we believe that there will be opportunities for faculty to further their professional and personal development. We share this list for all faculty, recognizing that each faculty member has different professional, family and personal realities during this time

Consider taking advantage of some of the following personal, academic, educational, research, and scholarly opportunities for yourself and the department of Surgery

We ask that you commit to at least one of these activities in the personal row, and customize, based on your at-hospital work schedule, and plan to undertake activities in rows 2-5 in the weeks ahead

1. Personal A. Make time for family/friends (virtually if necessary)

B. Remember to exercise-move in a way that makes you happy!

C. Work on good nutrition choices

D. Consider or develop your Mindfulness practices: Headspace is now offering free subscription to all health care professionals (https://www.headspace.com/health-covid-19) — a great time to explore and try. Calm is another meditation and mindfulness resource that has developed free resources for this time. https://www.calm.com/blogtake-a-deep-breath

E. Expand your knowledge/skills - pick up a book you have always wanted to read; catch up on your journal reading; consider ways to enhance your work as a master clinician

F. Give yourself 15 min a day (at least) to do something that brings you joy

G. Reach out to your colleagues-both here and remotely-and find out how they are doing; whether there is anything you can do to help them. Sometimes that is as listening to them!

2. Professional/Academic $\quad$ A. Update your OSU Find-a-Doctor profile information (your 'front office'-https://onesource.osumc. edu/sites/Audience/Physicians/Pages/FindAdoctor.aspx)

B. Review you CV and refine its formatting and completeness. Also, consider updating your NIH Biosketch

C. Review the Department of Surgery P\&T document relevant to your track and career stage; the center for FAME will be hosting an upcoming virtual session on $P \& T$ (https://oaa.osu.

edu/sites/default/files/updloads/governance-documents/college-of-medicine/surgery/Suergery APT 2017-10-28.pdf). The COM APT toolbox can be found here https://medicine.osu.edu/faculty/promotion-and-tenure/apt-toolbox. Please contact Suzanne Knott with questions

D. Methods to document talks and posters presented at conferences that have been moved to online only, been postponed or that are being held but the faculty member cannot attend (apastyle.apa.org/blog/canceled-conferences)

E. Complete required training in Buckeye Learn (https//:buckeyelearn.osu.edu)

F. Complete OSU FD4ME (very relevant online faculty development modules) free to all OSU faculty-https://fd4me.osu.edu/

G. Complete on-line CME (SESAP, Selected Readings in General Surgery), or Clinical Congress Webcasts; many journals also have CME available including all members of the JAMA Network)

3.Research/Scholarship A. Complete manuscripts in progress; consider drafting an abstract on your work that can be submitted for future meeting; write a review article (if not in the works, consider collaborating with colleague(s) and submit a proposal to a favorite editor)

B. Work on new research or scholarly projects - consider virtual 'brainstorming' meetings with colleagues, collaborators, mentees

C. Start, complete, or update IRBs at Buck-IRB

D. Plan and write grant applications

E. Brainstorm how you can turn 'lessons learned' from management of your patients in this current crisis into a research project, review and/or commentary

4. Educational A. Review and improve your existing presentations

*particularly like this PSU Effective Presenter of Engineering \& Science section on Structure https://www.engr.psu. edu/speaking/STRUCTURE.html

*format advocated by Duarte in Slide:ology: https://www.duarte.com/books/slideology/; and for a short tutorial: https://www. huffpost.com/entry/presentation-tips-slideology_b_2278955

B. Develop innovative new curricula, lectures and other educational materials for medical students, residents and fellows. Who can you collaborate with right now? Can this be a scholarly activity?

*reach out to Amalia Cochran, VC Education, Alan Harzman, General Surgery Residency Program Director, or Phoenix Chen, Director of Education Research, with ideas or for input

C. Collaborate with divisional and departmental colleagues by sharing lectures, image libraries, Power Point presentations, and other materials for teaching purposes

5. Advocacy A. Consider advocating for the house of surgery, particularly during this difficult time:

https://www.facs.org/advocacy/surgeonsvoice

B. Reach out to a colleague or faculty member engaged in advocacy and explore what you can contribute. Michael Sutherland and Amalia Cochran have both been involved with advocacy through the ACS

member when care of the patient will not be able to be performed alone by the faculty member (e.g., Level 1 trauma activation)

c. Perform evaluation and consultation of patients without suspicion of COVID-19. d. Participate in the operative care of COVID-19 positive patients on whom their team is operating. Cases will proceed with expediency and care, focused primarily on the care of the patient and the safety of the team rather than teaching. 
Table 5 Chair of surgery communication plan during the COVID19 crisis

Departmental meetings:

- Bi-weekly COVID-19 email updates - sent on Tuesdays and Fridays

- Weekly WebEx "Chair Office Hours"-Thursdays at 5:15 p.m.

- Bi-monthly "All Hands" WebEx meeting - Tuesdays at 5 p.m.

- Resident WebEx "Happy Hours" with Program Director-Sundays at 5 p.m.

Other regular leadership meetings:

- Daily DoS briefings/WebEx meetings with chair and executive administrative team

- Bi-weekly WebEx meeting with chair and division directors

- Daily Ohio State University Wexner Medical Center WebEx Briefings

DoS, department of surgery

e. Take precautions and don PPE in keeping with current medical center guidelines for healthcare personnel in effect at the time. These include specific guidelines for aerosolizing procedures and the correct method of donning and doffing PPE.

2. Phase II: Acceleration and peak of COVID-19 patients In addition to guidance in Phase I, Residents shall:

a. Defer evaluation and consultation of COVID-19 positive patients to a faculty member when possible.

\section{Phase III: Peak to steady state of COVID-19 patients}

a. Same guidance as Phase II

4. Phase IV: Care of COVID-19 positive patients outside a traditional surgical consultant role

In addition to the guidance in Phase III, residents shall:

a. Be assigned to patient care duties as determined by the program director of general surgery, in consultation with the chair and DIO, which may include the medical care of COVID-19 positive patients who are ill but do not specifically require a surgical intervention.

b. Continue to practice under the direction of the faculty and seek assistance and guidance as needed.

\section{Supply Chain Issues}

One of the issues that has created the most stress among the faculty and trainees leading up to the surge was the availability of PPE. Policies were established early to help preserve PPE, particularly N95 masks, and to avoid overuse or waste when use was not warranted. Aerosolizing and high-risk procedures were identified and included oral-maxillofacial surgery, thoracic, surgery, and head/neck and dental interventions with the intent to disrupt or break the mucosal membrane in the oral cavity, airway, and/or pulmonary tract, and cause prolonged exposure to aerosolization and/or droplets. It was recommended that N95 masks only be used for these aerosolizing procedures. At the point of care, the default was for clinicians to proceed with an abundance of caution. Continuous messaging and reinforcement are needed to avoid excessive use of PPE early in the "curve." Part of preparations for the surge was to include updates from the medical director of supply chain (SB) to provide real time data regarding our PPE inventory, especially N95 masks.

Part of that messaging included the projections for PPE use through the surge that emphasized the need for conservation prior to the surge. While it is still fairly unique for a health system to have a medical director for their supply chain, it proved to be an important part of the messaging plan to clinicians and helped alleviate faculty stress regarding the PPE planning. For systems without a supply chain medical director, we strongly encourage some engagement from a supply chain representative to directly answer questions and address faculty around PPE issues.

\section{Planning for Back to Business}

Once non-essential surgical procedures were postponed, operative cases were placed in a "depot" to be rescheduled after normal operations resumed. New teleconsults that would eventually require surgery were similarly placed in a depot for the schedulers. This process has led to hundreds of cases accruing that will need to be scheduled when the operating room schedule returns to normal. This "second surge" of elective cases will present its own set of logistic challenges and requires early planning to facilitate care of surgical patients once the COVID-19 surge has passed. Adding operating room staff and extending operating room hours will likely be required to accommodate the large volume of cases that will be rescheduled after the crisis. Additionally, the supply chain must ensure that the necessary equipment and supplies will be available for elective cases after the crisis is over. Planning with perioperative leadership is needed to prepare for these cases. A key facet of this plan is reassuring current personnel of their worth and value when their specialized services may not be needed during the crisis planning and surge. These personnel, who performed admirably during "normal" times, will be called upon greatly in the "back to business" phase after the crisis. Our institution has developed redeployment and volunteer opportunities for several employees, as well as a disaster leave policy to retain key members of our team. 


\section{Summary}

During times of crisis such as the COVID-19 pandemic, surgeon leadership in the health system is critical. Major reorganization is required to manage all of the challenges imposed by COVID-19, and surgeon leaders are key stakeholders in developing and implementing these radical changes. Clear and frequent communication, flexibility, and careful planning are required to navigate these unchartered waters. While a sense of urgency is required, a thoughtful and collaborative approach among leaders at every level will result in practical guidelines and policies that will help the faculty to safely get through this unprecedented crisis.

\section{Compliance with Ethical Standards}

Conflict of Interest Dr. Brethauer receives consulting fees from GI Windows and speaking honoraria from Medtronic. Dr. Poulose receives research support from BD Interventional and Advanced Medical Solutions and salary support from Americas Hernia Society Quality Collaborative; Dr. Needleman has no conflicts of interest; Dr. Pawlik has no conflict of interest.

\section{References}

1. World Health Organization website at https://www.who.int/ emergencies/diseases/novel-coronavirus-2019/technical-guidance. Accessed April 6, 2020.

2. Holshue ML, DeBolt C, Lindquist S, et al. First case of 2019 novel coronavirus in the United States. N Engl J Med 2020; 382:929-936.

3. Centers for Disease Control website at https://www.cdc.gov/ coronavirus/2019-nCoV/index.html. Accessed April 6, 2020.

4. Society for Critical Care Medicine website at https://sccm.org/ covid19. Accessed April 2, 2020

Publisher's Note Springer Nature remains neutral with regard to jurisdictional claims in published maps and institutional affiliations. 